\title{
Introduction to the Online Games and Game Like Systems Minitrack
}

\author{
Kafui Monu \\ University of Northern British \\ Columbia \\ $\underline{\text { Kafui.monu@unbc.ca }}$
}

\author{
Paul Ralph \\ University of Auckland \\ p.ralph@auckland.ac.nz
}

\author{
Jacky Xi Zhang \\ Tianjin University \\ jackyzhang@tju.edu.cn
}

Approaching $\$ 100$ billion in revenue, gaming has become the premier entertainment industry in the $21^{\text {st }}$ century. With the innovation of online games, users are now connected with gamers and companies every day. The result of this connectivity are new online communities, developer interaction and business models. Moreover, the wide reach and societal effects of online games demands serious study of gaming phenomena.

Additionally, serious games and gamified work systems are increasingly changing how we work and trade online. For years, innovative organizations including Nike, SAP and many universities have used these game-like systems to encourage commerce, education and citizen science. The interaction between online connectivity and game-oriented design will yield interesting research results and societal change as the use of these systems becomes even more widespread.

The purpose of the Online Games and Game-like Systems minitrack is to provide a forum for researchers to discuss the design, use and impact of online games and game-like systems in various contexts. Topics discussed in previous years have included game adoption, the use of games in organizations, the psychology of online games, and the effects of online games on individuals and groups.

The theme of this year's papers is the design of online games; specifically focusing on how to make these games more appealing for a longer period. The papers approach this theme from two very different directions: One focuses on the technical aspects of games while the other investigates the feelings and perceptions games should generate to promote continuuing play.

The first paper (If You Let Them Build It, They Will Stay! An Empirical Study of Add-on Content and User Engagement by Irfan Kanat, Yili Hong, Bin Gu and Raghu Santanam) investigates a set of games to determine whether user-generated content (UGC) and developer generated content or (DGC) lead to more ongoing play. UGC are modifications and additions to games created by players, typically after the game is released. DGC (also called Downloadable Content, DLC) are additions made available to players by developers (usually for a price) after the initial release of the game. This is a hot topic amongst video game players and some feel that DGC is an exploitative business practice. The paper finds that both UGC and DGC can extend the period in which players interact with games. Interestingly, no specific differences between the effects of UGC or DGC were found.

The second paper (The Influence of Perceived Belonging on Massively Multiplayer Online RolePlaying Games by Claus-Peter Ernst) investigates the player feelings that are associated with continued play. Through a survey, the paper identifies that the feeling of perceived belonging engendered by an online game is an important reason why people continue to play it. The authors advise designers to use certain tools and mechanics such as cooperative quests to design this sense of belonging into online games.

These papers represent two different approaches to understanding how games should be designed to engage players. Yet, much more research is needed in this area.

Understanding gamified information systems, and developing better ones, demands understanding both game design and information system design. Game design research remains in its infancy, with limited compelling theory, while information system development has been plagued by pseudoscience and dogmatic adgherence to long-refuted conjectures. Research at the intersection of game design and information system development is therefore rich in both challenges and opportunities. 